\title{
T-jump study of calcium binding kinetics of calcium chelators
}

\section{Mohammad Naraghi}

Department of Membrane Biophysics, Max-Planck-Institute for Biophysical Chemistry, Göttingen, Germany

\begin{abstract}
Summary Recent experimental studies have investigated the kinetic competition between calcium chelators and the secretion apparatus at a fast central synapse. Simultaneously, mathematical modelling studies indicate the importance of a quantitative knowledge of the binding kinetics of the chelators in studying fast physiological processes operating on a millisecond time scale. Using the temperature-jump relaxation method, I have studied the in vitro kinetics of BisFura-2, Furaptra, Fluo-3, Calcium-Green-1, Calcium-Green-5N, Calcium-Orange-5N as well as EGTA, BAPTA and $\mathrm{H}$-EDTA in conditions which are identical to those implemented in our patch clamp recordings, i.e. $100-140 \mathrm{mM} \mathrm{CsCl}$, 20-40 mM Cs-HEPES, $8 \mathrm{mM} \mathrm{NaCl}, \mathrm{pH}=7.2$ at $22^{\circ} \mathrm{C}$. The results can be summarized as follows: all fluorescent indicators have on rates in the range of $10^{8}-10^{9} \mathrm{M}^{-1} \mathrm{~s}^{-1}$. They differ significantly with respect to their off-rates from each other according to their affinities, ranging from $100 \mathrm{~s}^{-1}$ up to $26000 \mathrm{~s}^{-1}$. BAPTA is kinetically almost indistinguishable from Fura-2. EGTA and H-EDTA have small binding rate constants for calcium in the range of $3 \times 10^{6} \mathrm{M}^{-1} \mathrm{~s}^{-1}$ since, at $\mathrm{pH} 7.20$, protons need to be dissociated from the chelators before they can bind calcium ions.
\end{abstract}

\section{INTRODUCTION}

Calcium chelators are used extensively to measure cytosolic free calcium concentration, to buffer calcium to a predefined level or even to photorelease calcium by exposing a photolabile chelator to UV light [1-5]. In most of these studies, one needs to know the binding affinities of the chelators for calcium, since it is assumed that the chelators are in equilibrium with calcium ions. Nevertheless, a knowledge of the binding and unbinding rate constants is important in kinetic studies investigating transient, calcium mediated physiological processes which operate on the same time scale as the mean reaction time for the binding of calcium to the chelators of interest, e.g. the calcium transient during an action

Received 8 April 1997

Revised 1 August 1997

Accepted 14 August 1997

Correspondence to: Mohammad Naraghi, Department of Membrane Biophysics, Max-Planck-Institute for Biophysical Chemistry, Am Fassberg 11. D-37070 Göttingen, Germany

Tel: +49551201 1297; Fax: +495512011688

E-mail: mnaragh@gwdg.de potential [6] or the calcium transient at the release site in a presynaptic terminal [7]. Under these conditions, the exogenously added chelators compete with the endogenous, i.e. cellular calcium buffer system for binding calcium ions and represent a 'ow-pass filter' operating on the signal. Clearly, the extent to which each exogenous calcium buffer will be able to compete with the endogenous buffer system heavily depends on the speed of interaction of the buffers with calcium and, of course, on spatial distribution and mobility of the buffers. If highly localized interactions between calcium ions and various buffer systems are essential for the investigated physiological process, e.g. in synaptic transmission, then the problem involves another dimension because the spatial diffusional mobility and reaction act in concert to shape the calcium signals.

Whether one uses an indicator to observe rapid changes in free calcium concentration or adds a calcium buffer to bind a fraction of a transient calcium load, these considerations imply that one needs estimates of the binding rate constants in order to be able to predict the calcium time course faithfully. The first study to investigate the kinetics of a fluorescent calcium indicator was conducted by Kao and Tsien [8] who used the temperature-jump (T-jump) relaxation method $[9,10]$ to measure the binding kinetics 


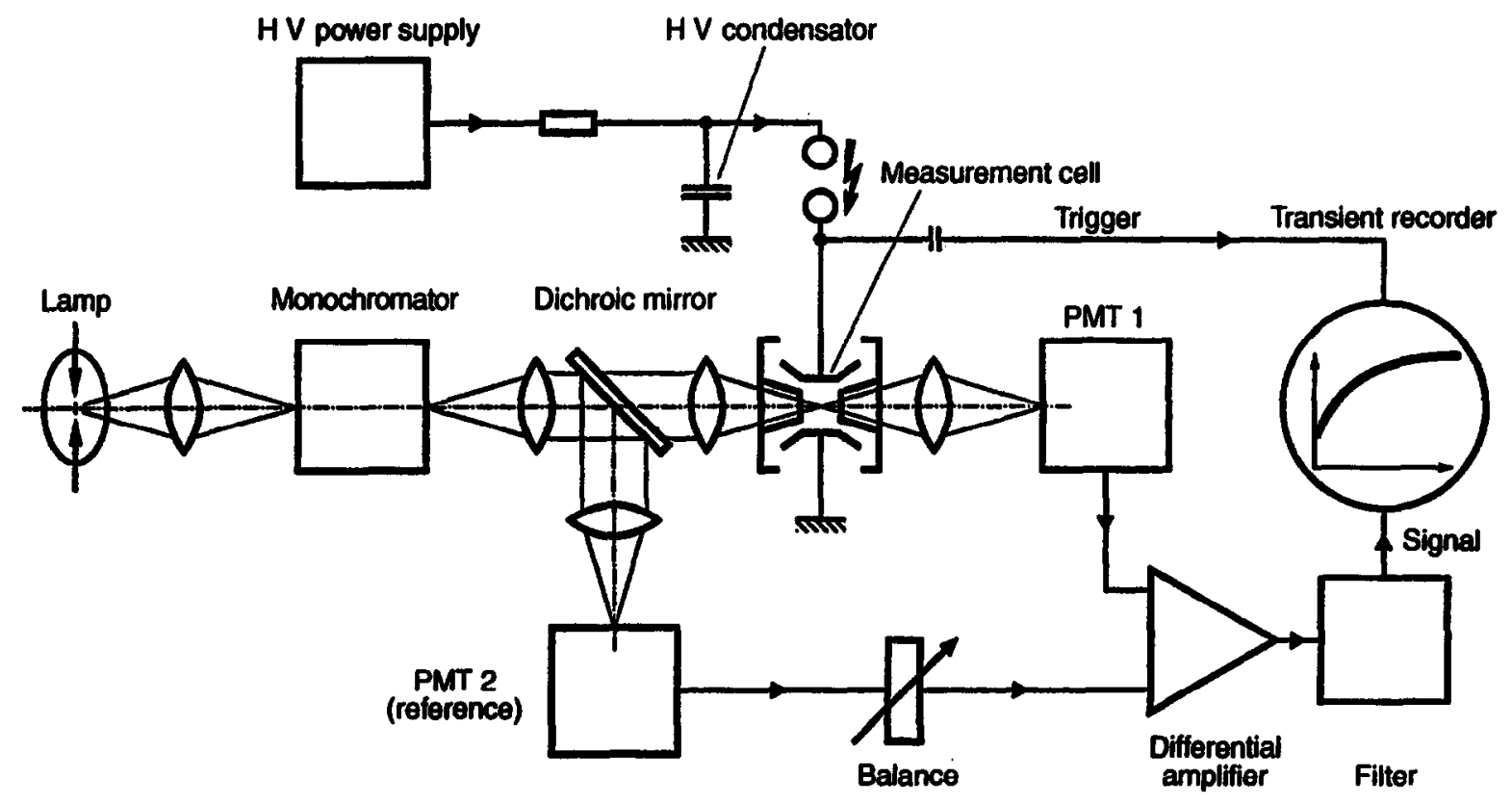

Fig. 1 Scheme of the T-jump setup for absorbance measurements. The light of a $600 \mathrm{~W} \times \mathrm{X}-\mathrm{Hg}$ arc lamp is passed through a monochromator to select the appropriate wavelength. A fixed fraction of this light is sent to the reference detector (PMT 2) to define the baseline and compared to the light intensity after passage through the sample as measured with PMT 1. A high voltage power supply is used to charge up the condensator which is discharged in the measurement cell after receiving a TTL high signal. The transient signal is recorded on a transient recorder after analog filtering, if necessary.

of Fura-2 and Azo-1. In the present study, the T-jump method is also used because of its high time resolution. A general treatment of the expected relaxation spectra for calcium binding to two competing buffer species is given in the Appendix. Some limiting cases of the results of the Appendix are used in the Results section for the analysis of the relaxation traces.

\section{MATERIALS AND METHODS}

For the preparation of the samples, we used twice distilled water which was, if necessary, treated with chelex to reduce the calcium contamination (see Results). The samples typically contained $8 \mathrm{mM} \mathrm{NaCl}$, between 20-40 mM HEPES, neutralized with $\mathrm{CsOH}$ and adjusted to $\mathrm{pH} 7.20,100-140 \mathrm{mM} \mathrm{CsCl}$ and different amounts of $\mathrm{CaCl}_{2}(1 \mathrm{M}$ certified stock solution from $\mathrm{BDH}$ Laboratory Supplies, Poole, UK).

For measuring kinetic properties of the fluorescent indicators (all from Molecular Probes Inc., Eugene, OR, USA), a high concentration (2-30 mM) of an appropriate non-fluorescent calcium chelator was used to buffer $\left[\mathrm{Ca}^{2+}\right]$ to values close to the dissociation constant of the indicator under study. In addition, around $10 \mu \mathrm{M}$ of the indicator was present to follow the progress of the relaxation by measuring the changes in the fluorescence of the indicator. BAPTA was approached similarly since its absorption spectra show marked changes in the molar extinction coefficient in the UV range between 240-300 $\mathrm{nm}$ upon binding of calcium. Thus, changes in UV absorption were monitored to follow the relaxation. In the case of EGTA and H-EDTA, the buffer was used itself to define $\left[\mathrm{Ca}^{2+}\right]$ while minute amounts of a fluorescent indicator were added to observe the relaxation upon temperature jump. All samples were filtered through a filter with pore size of $0.2 \mu \mathrm{m}$ and debubbled to remove dissolved gas which could otherwise generate bubbles in the sample cell upon rapid temperature change. The discharge circuit had typically resistances in the range of $150-200 \Omega$.

The T-jump apparatus [11] was manufactured by Garching Instrumente (Garching, Germany) and is schematically depicted in Figure 1. Briefly, the light source was a $600 \mathrm{~W} \mathrm{Xe-Hg} \mathrm{arc} \mathrm{lamp} \mathrm{the} \mathrm{light} \mathrm{of} \mathrm{which}$ was passed through a monochromator to give the appropriate wavelength with a bandwidth of $6 \mathrm{~nm}$. The temperature jump was achieved by discharging a $50 \mathrm{nF}$ capacitor which gave heating times in the range of 4-5 $\mu \mathrm{s}$. The samples were held at either $17^{\circ} \mathrm{C}$ or $18.5^{\circ} \mathrm{C}$ depending on whether the capacitor was charged to 26 $\mathrm{kV}$ or $22 \mathrm{kV}$. In both cases, the discharge resulted in a sample temperature of $22^{\circ} \mathrm{C}$. All experiments were repeated at least 5-10 times with a jump every $5 \mathrm{~min}$ to allow for the equilibration of the cell temperature to its 
prejump level. The relaxation traces were digitized with 10 bits as 4096 time points on a transient recorder (Biomation Cupertino, CA, USA). Subsequently, they were transferred to an IBM Unix workstation and analyzed by fitting routines kindly provided by $D$. Poerschke. The dissociation constants of BAPTA, H-EDTA, EGTA and DPTA were from C. Heinemann (personal communication).

\section{Calculation of the free calcium concentration}

Equation $\mathrm{A} 9$ in the Appendix clearly demonstrates that the relaxation rates will depend on the free calcium concentration after the jump, $\overline{\left[\mathrm{Ca}^{2+}\right]}$. In preparing the samples for the experiments, we always added known amounts of $\mathrm{A}, \mathrm{B}$ and $\mathrm{CaCl}_{2}$, i.e. $[\mathrm{A}]_{\mathrm{T}},[\mathrm{B}]_{\mathrm{T}}$ and $[\mathrm{Ca}]_{\mathrm{T}}$ were known. From the knowledge of these quantities and the affinities of the buffers $A$ and $B$ for calcium, $\overline{\left[\mathrm{Ca}^{2+}\right]}$ needs to be computed. For this purpose, assume a general system of the form:

$$
\mathrm{Ca}^{2+}+\mathrm{A}_{i} \underset{\mathrm{k}_{-\mathrm{A}_{i}}}{\stackrel{\mathrm{k}_{\mathrm{A}_{i}}}{\leftrightarrows}} \mathrm{CaA}
$$

Eq. 1

for $\mathrm{i}=1, \ldots, \mathrm{N}$. At equilibrium, the difference

$$
\mathrm{R}\left(\left[\mathrm{Ca}^{2+}\right]\right) \equiv[\mathrm{Ca}]_{\top}-\left[\mathrm{Ca}^{2+}\right]-\Sigma[\mathrm{CaA}]
$$

must be zero. Thus, we can compute $\overline{\left[\mathrm{Ca}^{2+}\right]}$ by searching for the zero of the objective functional $\mathrm{R}\left(\left[\mathrm{Ca}^{2+}\right]\right)$. It can be computed efficiently using the iterative NewtonRaphson algorithm [12]: let $\left[\mathrm{Ca}^{2+}\right]^{\mathrm{k}}$ denote the calculated calcium concentration at the $\mathrm{k}$-th step of the algorithm. Then, we set

$$
\begin{aligned}
{\left[\mathrm{Ca}^{2+}\right]^{k+1} } & =\left[\mathrm{Ca}^{2+}\right]^{k}-\frac{R\left(\left[\mathrm{Ca}^{2+}\right]^{k}\right)}{\frac{d R\left(\left[\mathrm{Ca}^{2+}\right]^{k}\right)}{d\left[\mathrm{Ca}^{2+}\right]}} \\
& =\left[\mathrm{Ca}^{2+}\right]^{k}+\frac{R\left(\left[\mathrm{Ca}^{2+}\right]^{k}\right)}{1+\sum_{i} \mathrm{~K}_{i}\left(\left[\mathrm{Ca}^{2+}\right]^{k}\right)}
\end{aligned}
$$

with

$$
K_{i}\left(\left[\mathrm{Ca}^{2+}\right]^{k}\right) \equiv \frac{\left[\mathrm{A}_{i}\right]_{\mathrm{T}} K_{A_{j}}}{\left(K_{A_{i}}+\left[C a^{2+}\right]^{k}\right)^{2}}
$$

being the calcium binding ratio of the $\mathrm{i}$-th buffer at the free calcium concentration $\left[\mathrm{Ca}^{2+}\right]^{k}[13]$. In the last identity, we have used

$$
[\overline{\mathrm{CaA}}]=\frac{[\mathrm{A}] \ldots: \overline{\left[\mathrm{Ca}^{2+}\right]}}{\left(\mathrm{K}_{\mathrm{A}_{\mathrm{f}}}+\left[\overline{\left.\mathrm{Ca}^{2+}\right]}\right)\right.}
$$

and differentiated this with respect to $\overline{\left[\mathrm{Ca}^{2+}\right]}$ to obtain the binding ratios $\kappa_{i}$. The initial condition is simply $\left[\mathrm{Ca}^{2+}\right]^{0}=$ 0 . We stop the iteration if the error condition $R\left(\left[\mathrm{Ca}^{2+}\right]^{k}\right) \leq$ $10^{-9} \mathrm{M}$ is satisfied, which is generally achieved within less than 10 iterations. The input parameters are the number of different species $N$, the total concentrations $\left[\mathrm{A}_{\mathrm{i}}\right]_{\mathrm{T}}$ and the dissociation constants $\mathrm{K}_{\mathrm{Aj}}$. All calculations are performed on a Macintosh Quadra 9500 computer using Matlab (MathWorks Inc., Natick, MA, USA).

\section{Deconvolution procedure for the analysis of Furaptra data}

Assume, we are looking at a chemical relaxation signal, $s(t)$, which is baseline subtracted and can be represented theoretically as a multiexponential process according to:

$$
s(t)=\sum_{k=1}^{n} a_{k}\left(1-e^{-v t_{k}}\right)
$$

with $\mathrm{n}$ different time constants $\tau_{\mathrm{k}}$. The actual measurement, however, is also affected by the response properties of the measurement system. These are the speed at which the temperature jump can be induced to perturb the equilibrium as well as the response time of the amplifier and detection system. Let us denote by $T(t)$ the time course of the temperature jump and by $\mathrm{D}(\mathrm{t})$ the impulse response of the detection system. Then, it is well known that the response function, $F(t)$, of the measurement apparatus is given by the convolution product

$$
F(t)=\left(T^{*} D\right)(t) \equiv \int_{-\infty}^{t} T\left(t^{\prime}\right) D\left(t-t^{\prime}\right) d t \quad \text { Eq. } 4
$$

and the actually measured relaxation signal, $s_{\text {obs }}(t)$, is itself the convolution product of $F(t)$ with $s(t)$, i.e. $s_{\text {obs }}(t)=$ $\left(F^{*} s\right)(t)$. In general, $F(t)$, can be a quite complicated function of time. If, however, $F(t)$ can be approximated by a sum of exponentials, the computation of the product $s_{\text {obs }}(t)$ is significantly simplified. In particular, if $F(t)$ is an exponential with the time constant $\tau$, the measured signal $s_{\text {obs }}(t)$ is given by

$$
\mathrm{s}_{\mathrm{obs}}(\mathrm{t})=\sum_{\mathrm{k}=1}^{n} \mathrm{a}_{\mathrm{k}}\left(1+\frac{\tau_{k}}{\tau-\tau_{k}} \mathrm{e}^{-\tau_{\mathrm{i}}}-\frac{\tau}{\tau-\tau_{k}} \mathrm{e}^{-v_{\tau}}\right) \quad \text { Eq. } 5
$$

in closed form. If the apparatus has a large bandwidth, i.e.

$$
\tau<\min _{k} \tau_{k}
$$

then $s(t)=s_{\text {obs }}(t)$. Otherwise, one has to explicitly use Equation 5 and least-squares routines to fit it to the observed data, assuming $\tau$ is known in advance. The latter can be achieved easily by measuring the response function, $F(t)$, of the system using a chemical relaxation process which is known to be fast compared with $F(t)$, i.e. 
if we use a chemical system where

$$
\max _{\mathrm{k}} \tau_{\mathrm{k}}<<
$$

is valid. Then, $s_{o b s}(t)=A+B e^{-t / r}$ for some constants $A$ and $B$ which gives us the information about $\tau$. For the analysis of Furaptra kinetics, we measure the response function $F(t)$ using absorbance changes of $5^{\prime}$-CMP.

\section{RESULTS}

\section{Indicators}

As mentioned in the previous section, we set $\left[\overline{\left.\mathrm{Ca}^{2+}\right]}\right.$, i.e. the free calcium concentration in the sample after the temperature jump, to different levels by using high concentrations of a second buffer with known affinity for calcium. Having also the indicator present in the sample (at a 100-1000-fold smaller concentration), the relaxation spectrum consists of two rate constants given by Equation A9. If, however, the buffer system is approaching its new equilibrium after the jump much faster than the indicator system $\left(\lambda_{1} \gg \lambda_{2}\right.$ in the notation of the Appendix), which can be achieved by increasing the concentration of the buffer, the smaller relaxation rate in Equation $A 9$ is given simply by

$$
\left.\lambda=k_{\mathrm{A}} \times \overline{\left[\mathrm{Ca}^{2+}\right.}\right]+k_{-A}
$$

Eq. 6

where $k_{A}$ and $k_{-A}$ represent the on- and off-rate of the indicator. Hence, a plot of $\lambda$ over $\overline{\left[\mathrm{Ca}^{2+}\right]}$ should give a line with the slope given by $k_{A}$ and the $y$-intercept $k_{-A}$.

\section{Bis-Fura-2}

Bis-Fura-2 is a new tetracarboxylate analogue of Fura-2 which has two main advantages over the classical calcium indicator Fura-2. It has two fluorophores attached to one calcium binding site. In addition, its dissociation constant for calcium is twice that of Fura-2. Hence, one would expect a higher fluorescent gain which in turn permits lower dye concentrations to be used in order to achieve a certain signal-to-noise ratio in noise-sensitive intracellular calcium measuring experiments.

We have used $\mathrm{Cs}_{4}-\mathrm{BAPTA}$ to clamp the $\overline{\left[\mathrm{Ca}^{2+}\right]}$ after the jump to predefined levels, using a dissociation constant of $220 \mathrm{nM}$ at $22^{\circ} \mathrm{C}$ and $\mathrm{pH}=7.2$. Figure $2 \mathrm{~A}$ demonstrates a typical relaxation trace which is fitted by a monoexponential curve. In theory, one has two relaxation rates given by Equation $A 9$. Figure $2 B$ shows why we nevertheless only see a monoexponential relaxation. We have plotted the two theoretically expected relaxation rate constants as a function of $\left[\overline{\left.\mathrm{Ca}^{2+}\right]}\right.$ for the conditions of our measurements. Clearly, there is a 1000 -fold difference between them. One process is relaxing on a $\mu$ s time scale, the other on a ms time scale. Hence, we expect to
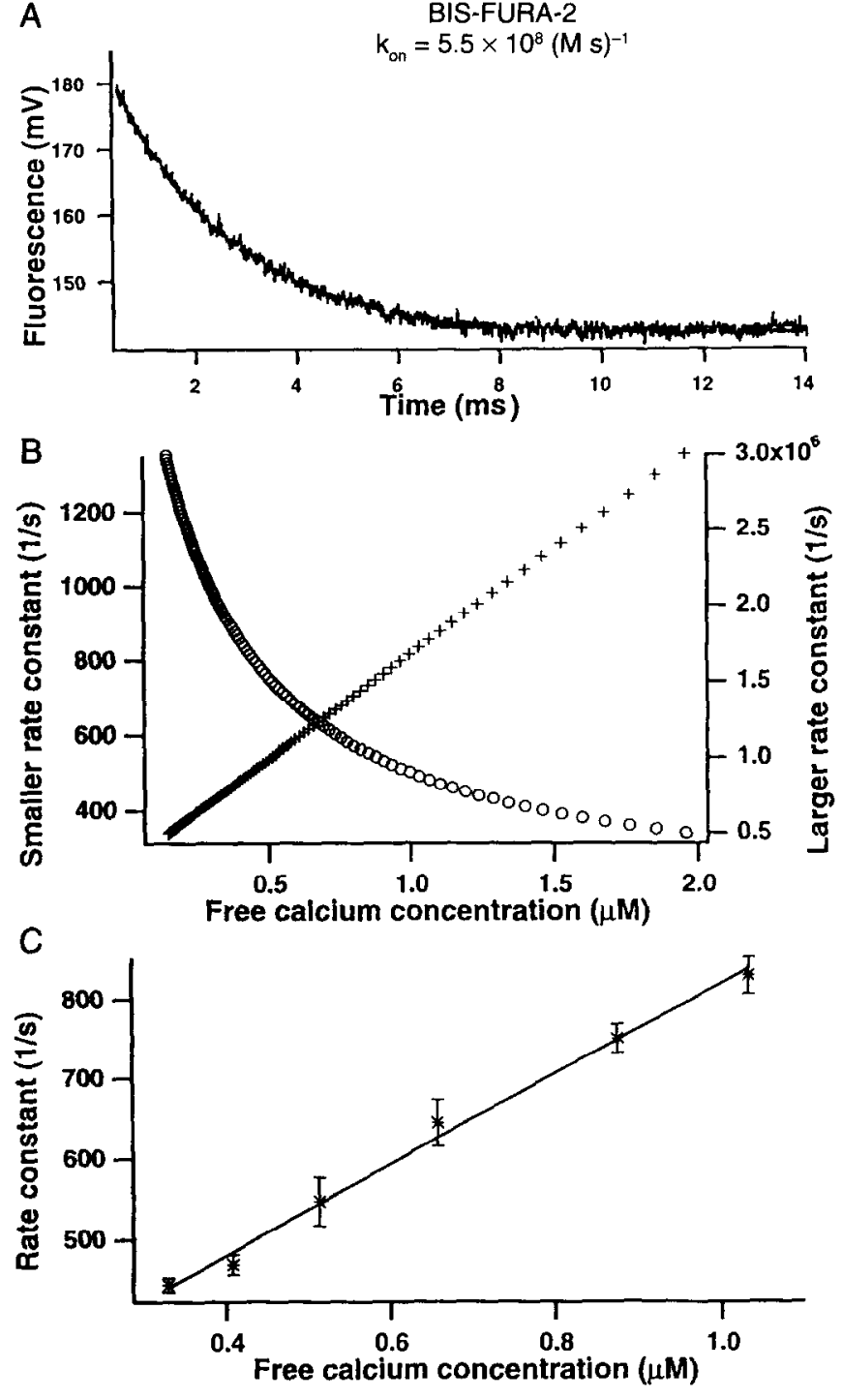

Fig. 2 Kinetics of Bis-Fura-2. The samples contained $10 \mu \mathrm{M}$ BisFura-2; $\left[\mathrm{Ca}^{2+}\right]$ was set by $10 \mathrm{mM} \mathrm{BAPTA}$ and $6.0-8.25 \mathrm{mM} \mathrm{CaCl}_{2}$. (A) shows a typical relaxation trace with a superimposed, monoexponential fit which gives a rate of $450 \mathrm{~s}^{-1}$. In this case, we had $\left[\mathrm{Ca}^{2+}\right]=330 \mathrm{nM}$. (B) is a plot of the two relaxation rate constants, $\lambda_{1}$ and $\lambda_{2}$, according to Equation $A 9$ as a function of $\left[\mathrm{Ca}^{2+}\right]$. The circles represent the larger rate constants whereas the crosses are the smaller rate constants. The smaller rates are a linear function of $\left[\mathrm{Ca}^{2+}\right.$ (in accordance with Eq. 6) while the larger rates depend non-linearly on $\left[\mathrm{Ca}^{2+}\right]$ and are beyond the resolution of our setup. (C) summarizes the Bis-Fura-2 data by depicting the average relaxation rates at different free calcium concentrations. A linear fit gives a slope of $5.5 \times 10^{8} \mathrm{M}^{-1} \mathrm{~s}^{-1}$ and a $y$-axis intercept of $257 \mathrm{~s}^{-1}$, corresponding to the on- and off-rate, respectively.

see the slower process with a linear dependence of $\lambda$ on $\left[\overline{\left.\mathrm{Ca}^{2+}\right]}\right.$ according to Equation 6 . Figure $2 \mathrm{C}$ shows that this is indeed the case. A linear fit gives an on-rate of $5.5 \times 10^{8}$ $\mathrm{M}^{-1} \mathrm{~S}^{-1}$ and an off-rate of $257 \mathrm{~s}^{-1}$. The on-rate is in good agreement with that of Fura-2 and implies that the 
introduction of the second fluorophore affects the affinity of the dye by increasing the rate of dissociation of calcium ions from the chelator.

\section{BAPTA}

BAPTA is a well known calcium chelator which is used extensively in physiology to buffer calcium in cells. It has the main advantage over its competitor, EGTA, that its affinity for calcium is much less $\mathrm{pH}$ dependent in the $\mathrm{pH}$ range around 7.0. Unlike EGTA, its calcium binding sites are mostly non-protonated at neutral $\mathrm{pH}$. This also implies that the speed of interaction with calcium should be higher than EGTA since no protons need to dissociate from BAPTA before calcium can bind to it.

BAPTA is hardly fluorescent. Nevertheless, we treat BAPTA in this section because its absorbance between $240 \mathrm{~nm}$ and $300 \mathrm{~nm}$ shows marked changes upon calcium binding, i.e. it can act as an absorbance calcium indicator [14]. Figure $3 \mathrm{~A}$ shows that the molar extinction coefficients change significantly by binding to calcium. Hence, we can follow the same strategy as before, but now monitor the changes in absorbance of the sample after a temperature jump. The background buffer for defining $\left[\overline{\left.\mathrm{Ca}^{2+}\right]}\right.$ was H-EDTA with a dissociation constant of $4.5 \mu \mathrm{M}$ at $22^{\circ} \mathrm{C}$ and $\mathrm{pH} 7.2$. Figure $3 \mathrm{~B}$ shows a typical relaxation trace which can be fitted with one exponential quite well. Figure $3 \mathrm{C}$ summarizes the data where we have titrated the relaxation rate constant over the free calcium concentration. Again, we see a nice linear dependence, as expected. A linear least squares fit gives us a slope of $4.5 \times 10^{8} \mathrm{M}^{-1} \mathrm{~s}^{-1}$ and a y-intercept of $79 \mathrm{~s}^{-1}$.

\section{Fluo-3, Calcium-Green-1, Calcium-Green-5N, Calcium- Orange- $5 \mathrm{~N}$}

In studies employing confocal microscopes, laser limitations often restrict the calcium indicator to be excitable by visible light. The currently available long wavelength indicators are usually excited by blue to red light and differ from each other by their equilibrium affinity for calcium. This makes them differentially suitable for measuring free calcium depending on the expected range of concentration one has to face.

In the case of high affinity indicators, Calcium-Green-1 and Fluo-3, $\overline{\left.\mathrm{Ca}^{2+}\right]}$ was set by using $5 \mathrm{mM}$ BAPTA; for Calcium-Green-5N and Calcium-Orange- $5 \mathrm{~N}, 10-20 \mathrm{mM}$ DPTA with a $K_{D}$ value of $80 \mu \mathrm{M}$ (under our experimental conditions) was used. Figure 4 summarizes the data for Fluo-3 (for brevity, no data are shown here for the other three indicators). Figure $4 \mathrm{~A}$ shows a typical relaxation trace of Fluo-3 with a monoexponential fit whereas Figure $4 \mathrm{~B}$ shows that the relaxation rate constant is indeed a linear function of the free calcium concentration after the jump. Fluo- 3 has an on-rate for calcium binding of $7.1 \times$ $10^{8} \mathrm{M}^{-1} \mathrm{~s}^{-1}$ and an off-rate of $369 \mathrm{~s}^{-1}$. The on-rates for
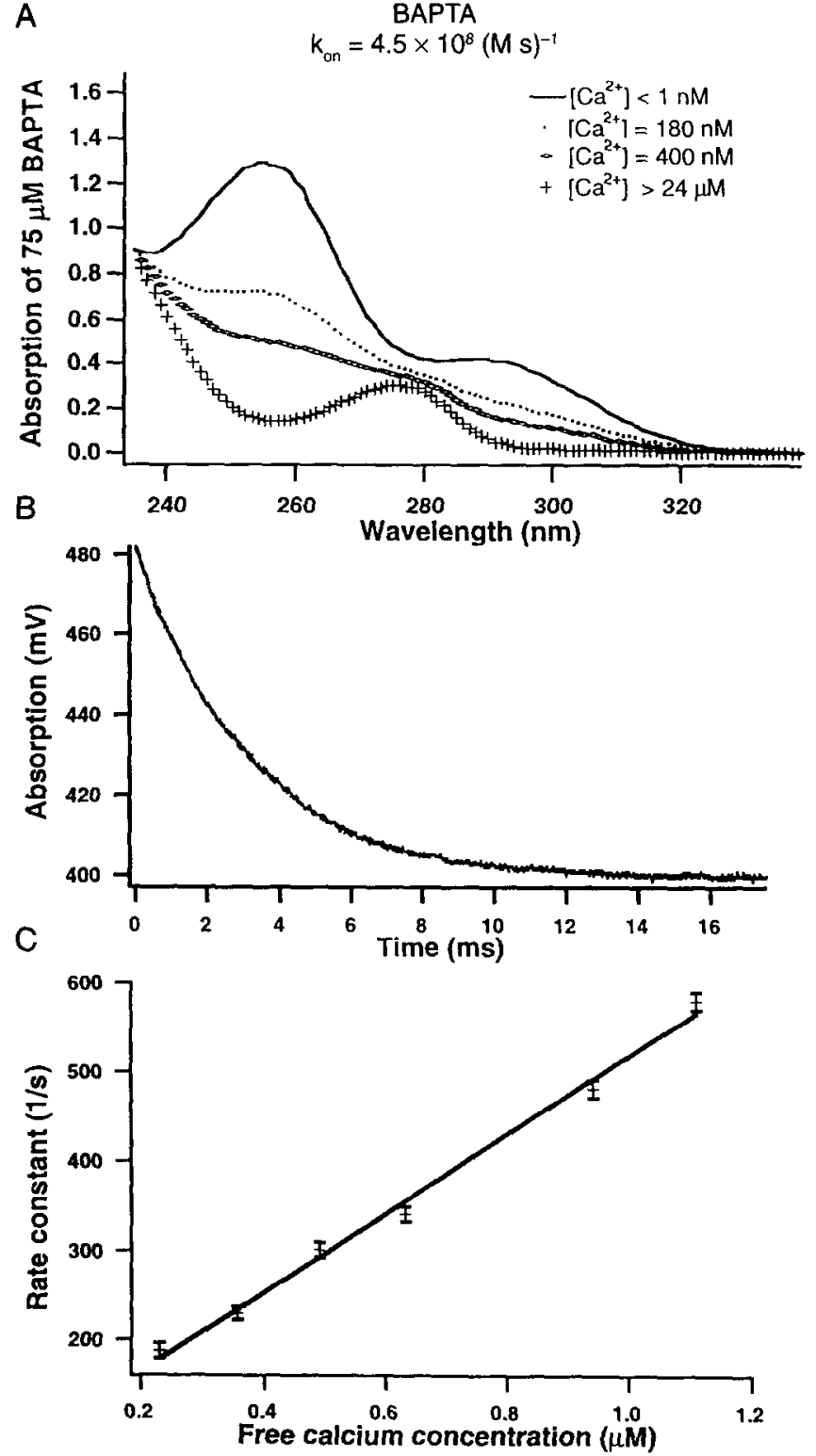

Fig. 3 Kinetics of BAPTA. For the T-jump measurements, the samples contained $50 \mu \mathrm{M}$ BAPTA; $20 \mathrm{mM} \mathrm{H}$-EDTA and 1.0-4.0 $\mathrm{mM} \mathrm{CaCl}$ were used to define $\left[\mathrm{Ca}^{2+}\right.$. We used the absorbance change of BAPTA (at $254 \mathrm{~nm}$ ) upon caicium binding to follow the relaxation process. (A) shows absorbance spectra of $75 \mu \mathrm{M}$ BAPTA at different free calcium concentrations (set by EGTA) as indicated in the figure. Clearly, calcium binding to BAPTA can reduce its absorbance by a factor of 6 . (B) is a typical relaxation trace at $\left[\mathrm{Ca}^{2+}\right]=631 \mathrm{nM}$. The monoexponential fit gives a rate of $330 \mathrm{~s}^{-1}$. (C) summarizes the data on BAPTA by plotting the average relaxation rates over $\left[\mathrm{Ca}^{2+}\right]$. A linear fit according to Equation 6 gives $\mathrm{k}_{\text {on }}=4.5 \times 10^{8} \mathrm{M}^{-1} \cdot \mathrm{s}^{-1}$ and $\mathrm{k}_{\text {off }}=79 \mathrm{~s}^{-1}$.

Calcium-Green-1, Calcium-Green-5N and CalciumOrange- $5 \mathrm{~N}$ are $7.5 \times 10^{8} \mathrm{M}^{-1} \mathrm{~s}^{-1}, 4.0 \times 10^{8} \mathrm{M}^{-1} \mathrm{~s}^{-1}$ and $2.1 \times$ $10^{8} \mathrm{M}^{-1} \mathrm{~s}^{-1}$, respectively. Hence, these indicators agree with respect to their binding rate-constants within a factor of 
A

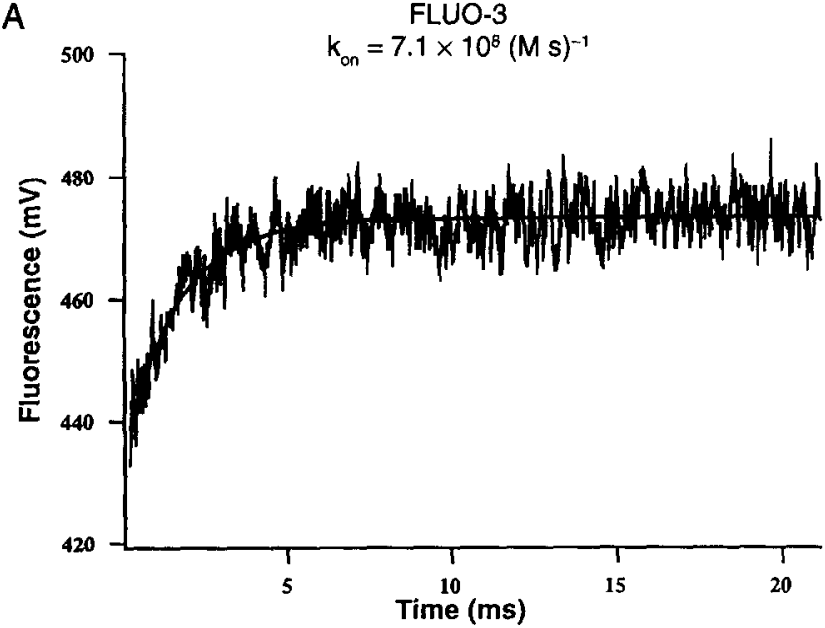

B

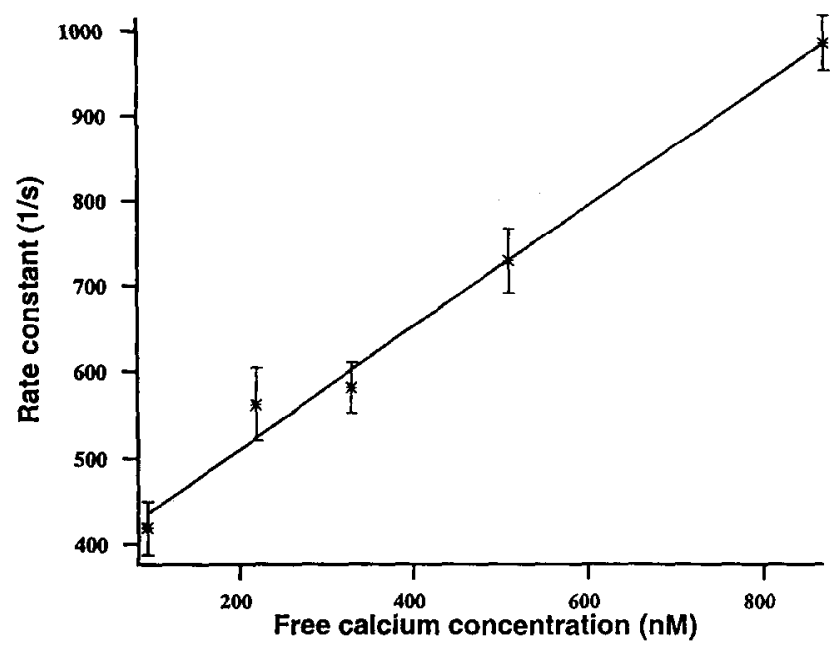

Fig. 4 Kinetics of Fluo-3. The solutions contained $5 \mu \mathrm{M}$ Fluo-3, 5 mM BAPTA and various amounts of $\mathrm{CaCl}_{2}$ to define $\left[\mathrm{Ca}^{2+}\right]$. (A) is a representative relaxation trace with a monoexponential fit. (B) depicts the average relaxation rates at different values of $\left[\mathrm{Ca}^{2+}\right]$ together with a linear fit according to Equation 6 . The estimated onrate is $7.1 \times 10^{8} \mathrm{M}^{-1} \mathrm{~s}^{-1}$ and the off-rate $369 \mathrm{~s}^{-1}$.

three. The off-rates, on the other hand, vary significantly depending on the indicator's affinity for calcium.

\section{Furaptra}

Furaptra is a tricarboxylate fluorescent indicator with a $\mathrm{K}_{\mathrm{D}}$ for calcium around $50 \mu \mathrm{M}$. Its main advantage over the indicators of the last section is the shift in the excitation spectrum it undergoes upon calcium binding. This property makes it ratiometric and hence appropriate for measurement of $\overline{\left[\mathrm{Ca}^{2+}\right]}$ in the tens of $\mu \mathrm{M}$ range. Many groups $[5,15]$ have used Furaptra to measure the dependence of the rate of calcium triggered secretion upon calcium concentration, using flash photolysis of DM-nitrophen to photorelease calcium. Because of the
A

FURAPTRA

$k_{\text {on }}=7.5 \times 10^{8}(\mathrm{M} \mathrm{s})^{-1}$
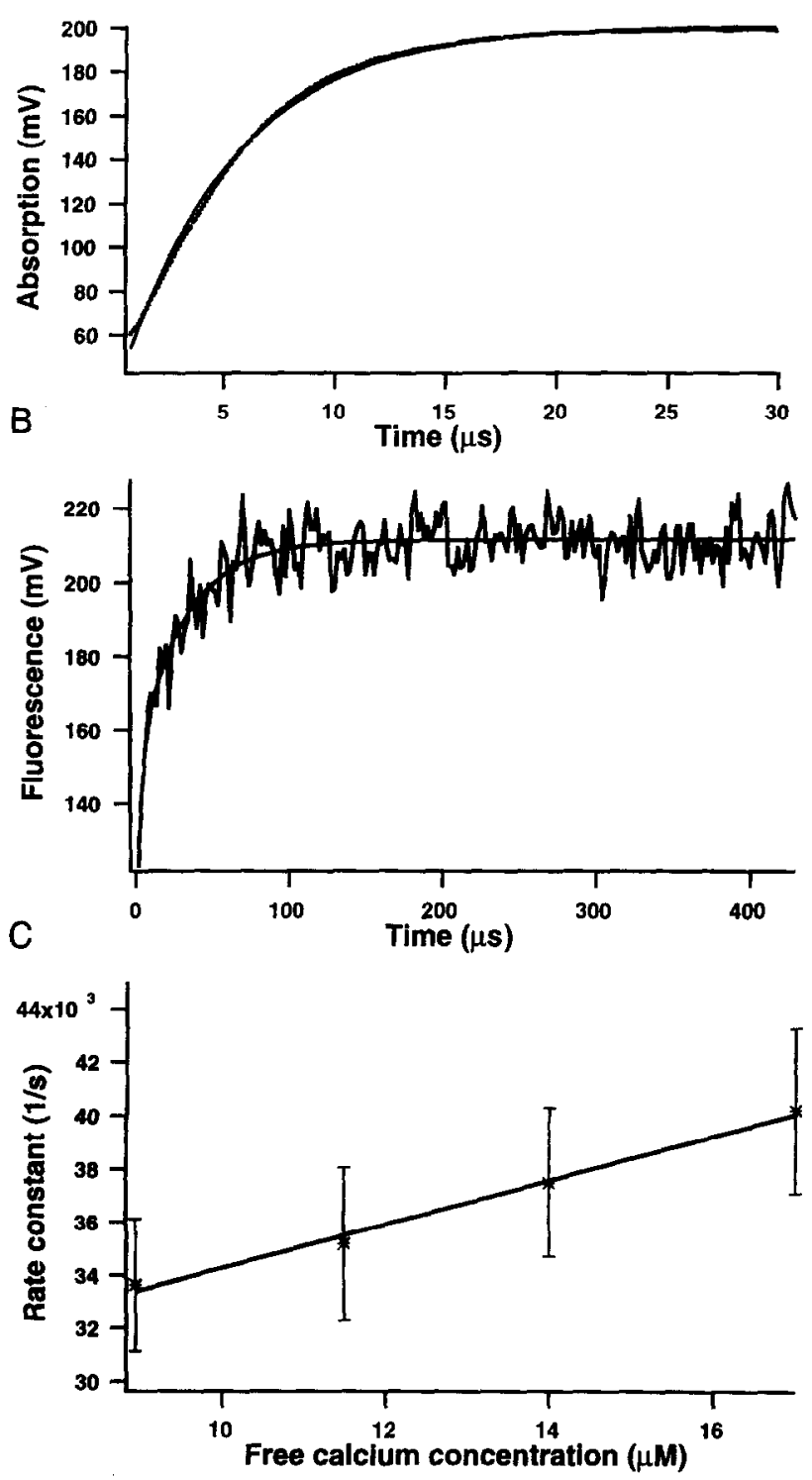

Fig. 5 Kinetics of Furaptra. (A) The response function, (see Eq. 4), of the T-jump apparatus was measured by observing the relaxation of the absorbance changes of $1 \mathrm{mM} \mathrm{5}$-CMP at $294 \mathrm{~nm}$. Superimposed is a fit with one exponential function which gives a time constant of $5.2 \mu \mathrm{s}$. In the Furaptra experiments, $\left[\mathrm{Ca}^{2+}\right]$ was set by $30 \mathrm{mM}$ DPTA and various amounts of $\mathrm{CaCl}_{2}$, using $20 \mu \mathrm{M}$ of the dye. (B) is a typical relaxation trace together with a fit according to Equation 5 . In (C), we have plotted the average relaxation rates as a function of $\left[\mathrm{Ca}^{2+}\right]$. A linear fit gives an on-rate of $7.5 \times 10^{8} \mathrm{M}^{-1} \mathrm{~s}^{-1}$ and an off-rate of $26760 \mathrm{~s}^{-1}$.

steep dependence of the secretion rate upon calcium, it is important to understand the calcium concentration time course after flash photolysis which in turn is determined by the binding and unbinding kinetics of the chelators present, in particular Furaptra. 
In studying the kinetics of Furaptra, we faced two difficulties: one was the signal-to-noise ratio of the relaxation signal and the other was the big relaxation rate corresponding to a time constant of around $30 \mu \mathrm{s}$ while the theoretical heating time constant was approximately $5 \mu \mathrm{s}$. The first problem was controlled by repeating the T-jump up to 50 times and averaging the relaxation signal. The second problem necessitated the use of deconvolution procedures which are described in 'Materials and methods'.

We have measured the response function, $F(t)$, of the apparatus (see 'Materials and methods' for notation) according to Poerschke [16] by measuring the relaxation of the absorbance signal of $1 \mathrm{mM} 5^{\prime}$-CMP (at $294 \mathrm{~nm}$ ) in the solution which we used for our T-jumps, using the same amplifier rise time as during the experiments with Furaptra. Under this condition, the chemical relaxation of $5^{\prime}$-CMP absorbance has a time constant in the range of tens of ns which is far below our expected heating time constant of many $\mu$ s. Thus, we can observe $F(t)$. Figure 5A shows a typical relaxation trace of 5'-CMP and demonstrates that $F(t)$ can be well fitted using one exponential with $\tau=5.2 \mu \mathrm{s}$. This finally justifies the use of Equation 5 to deduce the relaxation rate constant of calcium binding to Furaptra: we know $\tau$ and estimate $\tau_{k}$ by nonlinear fitting. In this way, one can avoid inverse deconvolution procedures which significantly amplify noise. Figure 5B shows a typical relaxation trace of Furaptra with a monoexponential fit according to Equation 5 with $n=1$, using the above value for $\tau$. Finally, Figure $5 \mathrm{C}$ summarizes the data where the relaxation rate constant $\lambda$ was titrated against $\left[\overline{\left.\mathrm{Ca}^{2+}\right]}\right.$ as in the previous sections to deduce the kinetic rate constants according to Equation 6. The linear fit gives an on-rate of $7.5 \times 10^{8}$ $\mathrm{M}^{-1} \mathrm{~S}^{-1}$ and an off-rate of $26760 \mathrm{~s}^{-1}$.

\section{EGTA, H-EDTA and DPTA}

For experiments employing kinetic competition between a cellular calcium sensor and an alien calcium buffer, one would like to have chelators with different properties, with high and low affinity as well as fast and slow kinetics. BAPTA as a fast high affinity buffer was characterized in the previous section. EGTA was investigated in the past with stopped-flow techniques $[17,18]$ and showed rather slow kinetics whereas there are no data to date on H-EDTA and DPTA.

The temperature-dependent change in the $\mathrm{pH}$ of HEPES-buffered solutions was used to drive the relaxation process since the equilibrium properties of the chelators are $\mathrm{pH}$-dependent. Free calcium concentration was set by the buffer under study whereas small amounts of Furaptra or Calcium-Orange-5N as low affinity and

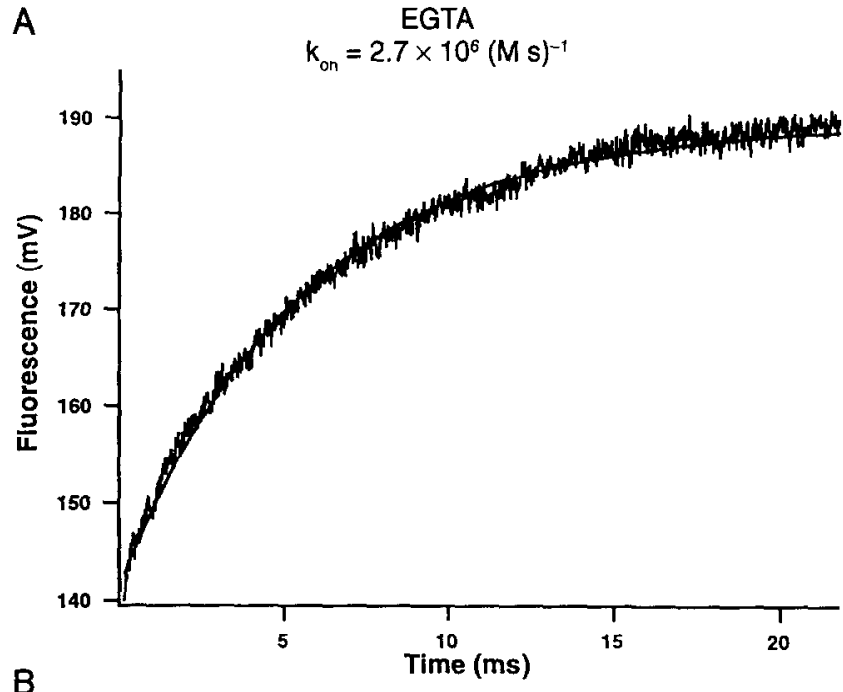

B

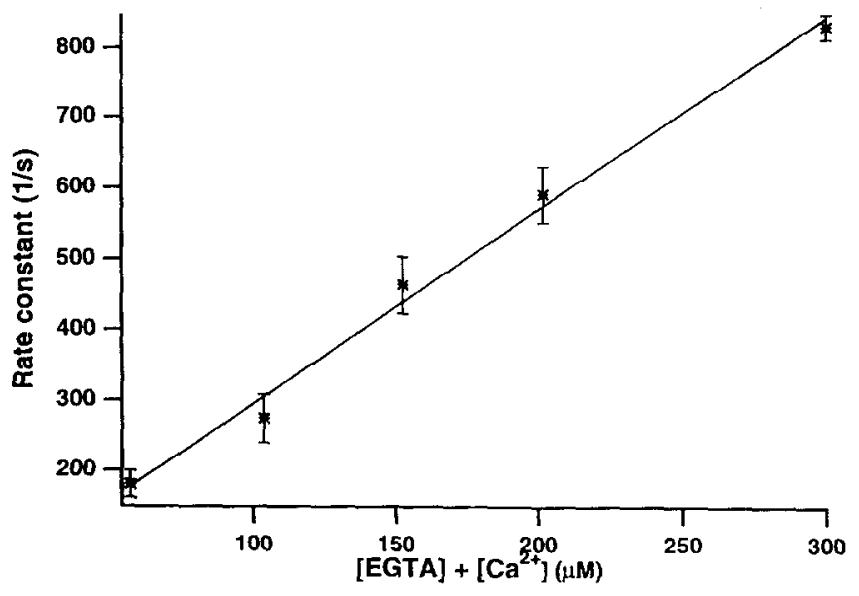

Fig. 6 Kinetics of EGTA. For studying EGTA, we used $20 \mu \mathrm{M}$ Furaptra as a low-affinity reporter of the relaxation process. $\left[\mathrm{Ca}^{2+}\right]$ was set by $1 \mathrm{mM}$ EGTA and various amounts of $\mathrm{CaCl}_{2}$. In (A), a typical relaxation trace is plotted together with an exponential fit. (B) plots the average relaxation rates over the sum [EGTA] + $\left[\mathrm{Ca}^{2+}\right]$. According to Equation 7, a line fit gives us an on-rate for EGTA of $2.7 \times 10^{6} \mathrm{M}^{-1} \mathrm{~s}^{-1}$. Since the off-rate is much smaller than $\mathrm{k}_{\text {on }}\left([\mathrm{EGTA}]+\left[\mathrm{Ca}^{2+}\right]\right)$, it was calculated as $\mathrm{k}_{\text {off }}=\mathrm{k}_{\mathrm{on}} \mathrm{K}_{0}$ to be $0.47 \mathrm{~s}^{-1}$.

fast calcium indicators were used to follow the relaxation spectrophotometrically.

Since we prefer to use low concentrations of the buffer, it is critical to avoid impurities in the buffer and the $\mathrm{CaCl}_{2}$ stock as far as possible. Using twice distilled water, we had a calcium background contamination of around $10-15 \mu \mathrm{M}$ in the solution consisting of $130 \mathrm{mM} \mathrm{CsCl}, 30$ $\mathrm{mM}$ HEPES and $8 \mathrm{mM} \mathrm{NaCl}(\mathrm{pH}=7.20)$ which was measured using Furaptra. After treating the twice distilled water twice with chelex, the same solution had a contamination of 1-2 $\mu \mathrm{M}$ which was considered as not significant since we had to add many hundreds of $\mu \mathrm{M}$ $\mathrm{CaCl}_{2}$ to the solution for setting $\overline{\left[\mathrm{Ca}^{2+}\right]}$. EGTA, H-EDTA 
and DPTA were heated for $90 \mathrm{~min}$ at $80^{\circ} \mathrm{C}$ to remove the water before making the solutions.

It is a classical result of relaxation kinetics [10] that, if a fast indicator such as Furaptra does not significantly bind calcium compared with the present buffer $B$, the reaction will proceed with a rate $\lambda$ given by:

$$
\left.\lambda=k_{B} \cdot\left(\overline{\left[\mathrm{Ca}^{2+}\right.}\right]+\overline{[B]}\right)+k_{-B}
$$

This indicates a strategy for estimating $k_{B}$ and $k_{-B}$ which is just a plot of $\lambda$ over $\overline{\left[\mathrm{Ca}^{2+}\right]}+\overline{[B]}$.

We used $1 \mathrm{mM}$ EGTA (with a dissociation constant of $179 \mathrm{nM}$ at $22^{\circ} \mathrm{C}$ and $\mathrm{pH}$ of 7.20 ) and various amounts of $\mathrm{CaCl}_{2}$ to define $\overline{\left[\mathrm{Ca}^{2+}\right]}$ after the jump. Figure $6 \mathrm{~A}$ shows a typical relaxation trace which is fitted with one exponential and Figure 6B summarizes the results of the titration of the relaxation rate over the sum $\left[\overline{\left.\mathrm{Ca}^{2+}\right]}+\right.$ $\overline{[\mathrm{EGTA}]}$. The slope of a line fit according to Equation 7 gives an on-rate of $2.7 \times 10^{6} \mathrm{M}^{-1} \mathrm{~s}^{-1}$. Under the conditions of our experiments, we have $\lambda \approx \mathrm{k}_{\mathrm{EGTA}} \cdot\left(\overline{\left[\mathrm{Ca}^{2+}\right]}+\overline{[\mathrm{EGTA}]}\right)$ which implies that the off-rate cannot be estimated faithfully from the linear fit. Hence, it was calculated as the product of the dissociation constant and the on-rate to be $0.47 \mathrm{~s}^{-1}$.

For the H-EDTA kinetics, we set the free calcium concentration with $1 \mathrm{mM} \mathrm{H}$-EDTA and various amounts of $\mathrm{CaCl}_{2}$ giving rise to $\overline{\left.\mathrm{Ca}^{2+}\right]}$ varying between $4.5 \mu \mathrm{M}$ and 34 $\mu \mathrm{M}$. The progress of the relaxation was monitored by sampling the fluorescence of $15 \mu \mathrm{M}$ Calcium-Orange-5N. Using the same approach as in the case of EGTA, one finally arrives at an on-rate of $4.5 \times 10^{6} \mathrm{M}^{-1} \mathrm{~s}^{-1}$. As before, the off-rate was estimated as the product of the dissociation constant at $22^{\circ} \mathrm{C}$ and $\mathrm{pH}$ of $7.20(4.5 \mu \mathrm{M})$ and the on-rate to be $20 \mathrm{~s}^{-1}$.

DPTA is a low affinity calcium chelator with a dissociation constant of $80 \mu \mathrm{M}$ (at $22^{\circ} \mathrm{C}$ and $\mathrm{pH}$ of 7.20) which has been used extensively to buffer calcium to concentrations in the tens of $\mu \mathrm{M}$ range, for instance in experiments employing flash photolysis of caged calcium to investigate the kinetics of secretion. Here, the question often arises whether there is a transient calcium spike after the flash (which could affect the time course of the secretory response of the cells). The answer, in turn, heavily depends on the kinetics of the present buffers which has prompted us to investigate the kinetics of calcium binding to DPTA.

Like BAPTA, DPTA changes its molar absorbance between $220 \mathrm{~nm}$ and $260 \mathrm{~nm}$ upon binding to calcium (data not shown here) but this change is rather small. We first made an attempt to buffer calcium with DPTA and monitor the relaxation after a temperature jump by observing this absorbance change. Unfortunately, this approach was not successful because of the small signal amplitude and its fast time course. The second approach
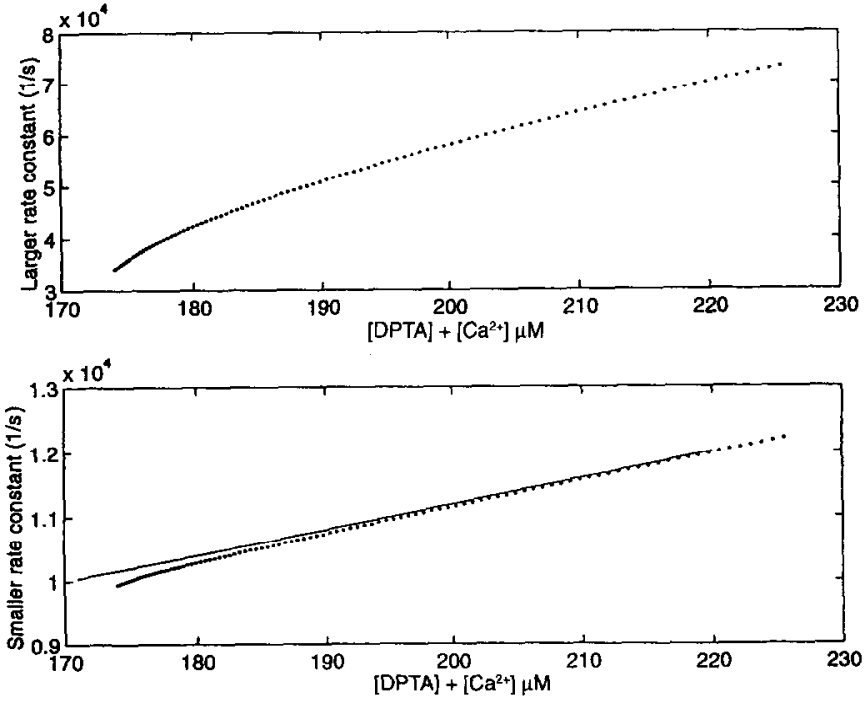

Fig. 7 Expected relaxation rate constants of Calcium-Green-5N fluorescence $(10 \mu \mathrm{M})$ in the presence of $200 \mu \mathrm{M}$ DPTA. For different concentrations of $\mathrm{CaCl}_{2}$, ranging from $150 \mu \mathrm{M}$ to $300 \mu \mathrm{M}$, $\left[\mathrm{Ca}^{2+}\right.$ and [DPTA] were calculated as well as the two rate constants according to Equation A9. These theoretical rate constants are plotted here as a function of $\left[\mathrm{Ca}^{2+}\right]+[\mathrm{DPTA}]$ (dotted lines). The solid line in the second panel is just the relationship $\lambda=$ $\mathrm{k}_{1} \times\left(\left[\mathrm{Ca}^{2+}\right]+[\mathrm{DPTA}]\right)+\mathrm{k}_{-1}$, with $\mathrm{k}_{1}=4.0 \times 10^{7} \mathrm{M}^{-1} \cdot \mathrm{s}^{-1}$ and $\mathrm{k}_{-1}=$ $3200 \mathrm{~s}^{-1}$. This demonstrates that we should have been able to extract the DPTA kinetics with the above on- and off-rates.

was to buffer calcium with DPTA and monitor the fluorescence or absorbance of a fast reporter dye, just as in the case of EGTA and H-EDTA. For this reason, we combined hundreds of $\mu \mathrm{M}$ DPTA with small amounts of Furaptra, murexid, tetramethylmurexid, CalciumGreen-5N or Calcium-Orange-5N. In the case of Furaptra, murexid and tetramethylmurexid, it was not possible to get a sufficiently high signal. In the case of the long wavelength fluorescent indicators, the DPTA kinetics were so fast that the kinetics of the indicators became ratelimiting, i.e. the observed rates were comparable to the relaxation rates of the indicators which acted like low-pass filters. This made it impossible to give precise estimates of the rate-constants using the fluorescent dyes. Thus, we can only give lower estimates for the on-rate of calcium binding to DPTA. We base this estimate on our observations with Calcium-Green-5N: the dotted lines in Figure 7 show the expected dependence of the two relaxation rates of the Calcium-Green- $5 \mathrm{~N}$ fluorescence (present at $10 \mu \mathrm{M}$ total concentration with on- and offrates as determined in this study) on $\left[\overline{\left.\mathrm{Ca}^{2+}\right]}+[\overline{\mathrm{DPTA}}]\right.$, with $150-300 \mu \mathrm{M} \mathrm{CaCl}_{2}$ which is buffered by $200 \mu \mathrm{M}$ DPTA, i.e. under our experimental conditions. Here, we have assumed DPTA to have an on-rate of $4.0 \times 10^{7} \mathrm{M}^{-1} \mathrm{~s}^{-1}$ and an off-rate of $3200 \mathrm{~s}^{-1}$. Clearly, there is a 3-6-fold difference between the two rates with the smaller one 
corresponding to $100 \mu$ s time constant. Thus, we would be able to see the slower process which reflects the DPTA kinetics. Indeed, the solid line in the second panel of Figure 7 is just a plot of the linear relationship $\lambda=k_{1} \times$ $\left(\left[\mathrm{Ca}^{2+}\right]+[\mathrm{DPTA}]\right)+\mathrm{k}_{-1}$, demonstrating that the kinetics of DPTA should be visible under these conditions. In our actual measurements, however, we get monoexponential relaxations with rate constants around $30000 \mathrm{~s}^{-1}$. This is the expected rate of Calcium-Green-5N relaxation if it was the rate-limiting factor. Calcium-Green- $5 \mathrm{~N}$ kinetics, in turn, only becomes rate-limiting if DPTA relaxation is too fast for Calcium-Green-5N to be able to report on it. This will happen with DPTA on-rates in the range of $1.0 \times$ $10^{8} \mathrm{M}^{-1} \mathrm{~s}^{-1}$ or bigger, but not with an on-rate of $4.0 \times 10^{7}$ $\mathrm{M}^{-1} \mathrm{~s}^{-1}$ as we saw before. The conclusion is: $\mathrm{k}_{1} \geq 4.0 \times 10^{7}$ $\mathrm{M}^{-1} \mathrm{~s}^{-1}$ and $\mathrm{k}_{-1} \geq 3200 \mathrm{~s}^{-1}$.

\section{Discussion}

In the last few years, evidence has accumulated supporting the idea that we should think about calcium signals as being sharply localized in time and space, at least in studying fast physiological processes [19-21]. In synaptic transmission, for instance, the delay between calcium influx through voltage-dependent channels and release can be as short as 200-300 $\mu$ s [22-24]. The implications of this are two-fold: (i) a close spatial proximity between the channels and the secretion apparatus is necessary to make the diffusional times short; and (ii) rapid binding of incoming calcium to the sensor responsible for triggering release is necessary to initiate exocytosis within a short delay. On the other hand, to achieve synchronous release with frequencies in the range of, say, $100 \mathrm{~Hz}$, calcium should rapidly dissociate from the sensor to reset the secretion apparatus before the next action potential invades the terminal. In ventricular cardiomyocytes, local interactions between calcium in a microdomain surrounding the mouth of L-type channels and surrounding channels or the adenylyl cyclase system has been implicated in modulating the channels and excitationcontraction coupling $[25,26]$. These studies indicate that local calcium signalling involves two dimensions: diffusional mobility of calcium (and other calciumcarrying species like chelators) as well as kinetics of binding and unbinding to cellular effectors (and chelators). Both aspects determine the temporal and spatial profiles of $\left[\mathrm{Ca}^{2+}\right]$ and, thus, the effects of a stimulus which excrts its influence using calcium as a highly controlled second messenger.

To infer information from observations of a dynamic system, one inevitably needs to manipulate it, i.e. to tune its parameters to different ranges. Correspondingly, the experimenter has two tools in his hands to manipulate the system in order to explore and dissect the differential effects of the determinants of fast calcium signals: (i) changing the effective calcium mobility by introducing chelators into the cell with different diffusional characteristics; or (ii) inducing a kinetic competition between a calcium chelator of known kinetics with a cellular one which needs to be characterized kinetically. The first method, although very useful in certain instances, has a rather limited utility despite the fact that dextran-coupled chclators are available. The diffusion coefficient (in water) grows with $\mathrm{M}^{-1 / 3}(\mathrm{M}=$ molecular weight of the chelator) and we do not have control over the chelator mobility in the cell. In addition, in a patch clamp experiment, loading a cell with a low-mobility chelator can take a prohibitively long time or even be impossible if one aims at small structures like, for instance, dendritic trees. This practically implies that one can reduce the apparent calcium diffusion coefficient by, at most, a factor of 10 . On the other hand, the 'kinetic competition' approach is quite promising, since the calcium binding kinetics of chelators can vary by $2-3$ orders of magnitude. It is this experimental freedom which points to the importance of a quantitative knowledge of buffer kinetics in studying rapid calcium signals. Furthermore, theory and simulations are providing us with additional insights regarding the importance of a chelator's on-rate in determining its ability to buffer calcium close to a channel mouth [27-30]. These studies have prompted us to determine the on- and off-rates of commonly employed chelators under conditions which match patch clamp recording situations, using relaxation methods. We have decided to approach this problem employing the T-jump relaxation technique [10] because of its superior time resolution.

In this paper, we give a general description of the expected relaxation rates (for two calcium buffers) as a function of equilibrium calcium concentration, $\left[\overline{\left.\mathrm{Ca}^{2+}\right]}\right.$, and the individual buffer's kinetic parameters. Like Kao and Tsien [8], we use the temperature dependence of the $\mathrm{pH}$ of HEPES-buffered solutions and, consequently, the $\mathrm{pH}$ dependence of the affinities of calcium buffers to induce a perturbation from the pre-jump equilibrium to the post-jump equilibrium. Two limiting cases must be distinguished.

Firstly, if the buffer which is to be characterized kinetically, say A in the scheme (Eq. A1), itself changes some optical property upon calcium binding, i.e. its absorbance or fluorescence, we pursue the following strategy: using a high concentration of a second buffer, $B$, with known affinity for calcium, we define the post-jump $\overline{\left[\mathrm{Ca}^{2+}\right]}$. Simultaneously, by increasing the concentration of this buffer to sufficiently high levels, we can make its relaxation time so short that it reaches its post-jump equilibrium instantaneously compared with the reaction time scales of $A$. Then, upon a T-jump, $\overline{\left[\mathrm{Ca}^{2+}\right]}$ 
instantaneously is set to its new value by $B$ and the buffer $A$ has to relax towards its new equilibrium given by $\left[\overline{\mathrm{Ca}^{2+}}\right]$. The progress of this reaction is followed by monitoring some optical property of $A$, i.e. by monitoring the change in [CaA]. This is a typical calcium-jump experiment and is used in the cases of Bis-Fura-2, BAPTA, Furaptra, Fluo-3, Calcium-Green-1, CalciumGreen-5N and Calcium-Orange-5N.

Secondly, if $A$ is optically inert, we add a minute concentration of a second buffer, $B$, which does change its absorbance or fluorescence upon calcium binding. Here, post-jump $\overline{\left[\mathrm{Ca}^{2+}\right]}$ is set to well-defined levels by a sufficiently high concentration of $A$ to make any calcium contamination insignificant. Using Van't Hoff's equation

$$
\begin{aligned}
\frac{\partial \ln K_{A}}{\partial T} & =\frac{\Delta H}{R T^{2}} \\
\text { (or } \quad \frac{\Delta K_{A}}{K_{A}} & =\frac{\Delta H}{R T^{2}} \Delta T \text { in its linearized version) }
\end{aligned}
$$

which describes the temperature-dependence of $K_{A^{\prime}}$ the affinity of $\mathrm{A}$ is changed as fast as $\mathrm{T}$ can be changed. Thus, after the T-jump, the free calcium concentration must relax to its new value determined by the post-jump value of $\mathrm{K}_{\mathrm{A}}$. In order for $\mathrm{B}$ to report on this relaxation, it must fulfil the condition of being fast, i.e. its relaxation time must be much shorter than that of $A$. Otherwise, one would see the kinetics of $B$ and not of $A$. But we cannot make the relaxation time of $B$ faster simply by increasing its concentration since, otherwise, it would contribute to calcium buffering and there would be no simple relationship between the observed $\lambda$ and the on- and offrates of $A$. To conclude, the essential criterion here is the availability of a fast reporter dye, $B$, which can be used at a minute concentration without significant calcium buffering. Given a choice of $B$, this also imposes an upper limit on the maximally tolerable concentration of $A$ since increasing $[A]_{T}$ eventually makes its relaxation time comparable to that of $B$ which is something we cannot afford. With these constraints in mind, this is the implemented approach for studying EGTA, H-EDTA and DPTA.

The Table summarizes the data regarding the on- and off-rates. Clearly, BAPTA and Bis-Fura-2 have similar onrates around $5.0 \times 10^{8} \mathrm{M}^{-1} \mathrm{~s}^{-1}$ and differ from each other with respect to their off-rates. For comparison, we also measured Fura-2 under the same ionic conditions and obtained for its on-rate a value of $4.0 \times 10^{8} \mathrm{M}^{-1} \mathrm{~s}^{-1}$ which is in good agreement with the data of Kao and Tsien [8], namely $6.0 \times 10^{8} \mathrm{M}^{-1} \mathrm{~s}^{-1}$. Indeed, they are all tetracarboxylate chelators. BAPTA has no fluorophore attached to its chelating group, Fura-2 has one whereas Bis-Fura-2 has two. Thus, the introduction of the fluorophores does not significantly interfere with the association kinetics. Instead, the second fluorophore in Bis-Fura-2 has doubled the calcium dissociation probability (per unit time) from the chelating group. This implies that the dye achieves equilibrium within $5 \mathrm{~ms}$ (at $1 \mu \mathrm{M}$ free calcium according to Eq. 6) if used in small concentrations to report on intracellular $\left[\mathrm{Ca}^{2+}\right]$, i.e. it can be used with this time resolution without significant kinetic low-pass filtering of the calcium signal. In addition, compared with Fura-2, the higher $\mathrm{K}_{\mathrm{D}}$ of $470 \mathrm{nM}$ reduces the equilibrium binding ratio ( $\mathrm{k}$ ) of the dye while the second fluorophore gives rise to a higher fluorescence gain (although the fluorescence increase is not a factor of 2 as one could optimally expect). This allows the use of smaller dye concentrations with better kinetic resolution and without loss of signal-to-noise ratio.

Furaptra is a tricarboxylate chelator which has the same fluorophore attached to it as Fura-2 and, hence, has similar spectral shifts. It has a lower affinity for

\begin{tabular}{|c|c|c|c|}
\hline Buffer & $\mathrm{k}_{\mathrm{on}}(1 /(\mathrm{M} . \mathrm{s}))$ & $k_{\text {off }}(1 / s)$ & $K_{\mathrm{D}}(\mu \mathrm{M})$ \\
\hline $\begin{array}{l}\text { Calcium-Green-1 } \\
\text { Fluo-3 } \\
\text { Calcium-Green-5N } \\
\text { Calcium-Orange-5N }\end{array}$ & $\begin{array}{l}(7.5 \pm 0.25) \times 10^{8} \\
(7.1 \pm 0.31) \times 10^{8} \\
(4.0 \pm 0.14) \times 10^{8} \\
(2.1 \pm 0.23) \times 10^{8}\end{array}$ & $\begin{aligned} 120 & \pm 12 \\
369 & \pm 25 \\
9259 & \pm 190 \\
11670 & \pm 273\end{aligned}$ & $\begin{array}{l}0.16^{\star} \\
0.52^{\star} \\
23.1^{\star} \\
55.6^{\star}\end{array}$ \\
\hline $\begin{array}{l}\text { Fura-2 } \\
\text { Bis-Fura-2 } \\
\text { Furaptra } \\
\text { BAPTA }\end{array}$ & $\begin{array}{l}(4.0 \pm 0.18) \times 10^{8} \\
(5.5 \pm 0.21) \times 10^{8} \\
(7.5 \pm 1.15) \times 10^{8} \\
(4.5 \pm 0.15) \times 10^{8}\end{array}$ & $\begin{aligned} 103 & \pm 7 \\
257 & \pm 14 \\
26760 & \pm 1800 \\
79 & \pm 4\end{aligned}$ & $\begin{array}{l}0.258^{\star} \\
0.467^{\star} \\
35.7^{\star} \\
0.176^{\star}\end{array}$ \\
\hline $\begin{array}{l}\text { EGTA } \\
\text { H-EDTA } \\
\text { DPTA }\end{array}$ & $\begin{array}{r}(2.7 \pm 0.25) \times 10^{6} \\
(4.5 \pm 0.27) \times 10^{6} \\
\geq 4.0 \times 10^{7}\end{array}$ & $\begin{array}{c}0.5^{\#} \\
20^{\#} \\
\geq 3200\end{array}$ & $\begin{array}{l}0.180^{+} \\
4.4^{+} \\
80^{+}\end{array}$ \\
\hline
\end{tabular}

Table Summary of the results

${ }^{*} K_{D}$ derived from kinetlc rate constants as $k_{-1} / k 1$

+ According to $C$. Heinemann (personal communication)

"Off-rate calculated as $\mathrm{k}_{1} \times \mathrm{K}_{\mathrm{D}}$. 
calcium and has been used for measuring $\left[\mathrm{Ca}^{2+}\right]$ in presynaptic nerve terminals or in skeletal muscle fibers $[5,6,23,31,32]$. A question intimately related to Furaptra kinetics in these studies is whether it is able to track the $\left[\mathrm{Ca}^{2+}\right]$ time course faithfully without any kinetic delay, either in response to an action potential or to flash photolysis of caged calcium. Zhao et al [6] give a lower limit for its on-rate $\left(>5.0 \times 10^{7} \mathrm{M}^{-1} \mathrm{~s}^{-1}\right)$ assuming that its myoplasmic $K_{\mathrm{D}}$ is $100 \mu \mathrm{M}$ and its off-rate at least $5000 \mathrm{~s}^{-1}$. Here, we report on its on- and off-rate: its on-rate is in the same range as the tetracarboxylate chelators (namely 7.5 $\times 10^{8} \mathrm{M}^{-1} \mathrm{~s}^{-1}$ ) while its off-rate is around $26700 \mathrm{~s}^{-1}$, corresponding to the high $\mathrm{K}_{\mathrm{D}}$ value. Thus, its relaxation time constant is shorter than $40 \mu$ s and it should be able to follow $\left[\mathrm{Ca}^{2+}\right]$ with a delay less than $150 \mu \mathrm{s}$.

The long-wavelength indicators studied here all have a tetracarboxylate chelating group. They seem to segregate in two groups: the low affinity indicators with on-rates between $2-4 \times 10^{8} \mathrm{M}^{-1} \mathrm{~s}^{-1}$ and the high affinity indicators with on-rates around $7.0 \times 10^{8} \mathrm{M}^{-1} \mathrm{~s}^{-1}$. A common feature is the observation that the number of negative charges on the dye positively correlates with its on-rate. In a study employing stopped-flow measurements to investigate the dissociation kinetics of calcium from Calcium-Green-1 [33], the authors estimate $\mathrm{k}_{-1}$ to be 176 $\mathrm{s}^{-1}$, at $\mathrm{pH} 7.20$ and ionic strength of $0.175 \mathrm{M}$ (temperature unclear), but no direct measurements of $k_{1}$ could be performed. Here, we determine $k_{1}$ and $k_{-1}$ independently. Our value for $\mathrm{k}_{-1}\left(120 \mathrm{~s}^{-1}\right)$ is $30 \%$ smaller than the above value. Two other studies $[34,35]$ have employed stoppedflow measurements to estimate $\mathbf{k}_{-1}$ for Fluo-3. For initial values of $\left[\mathrm{Ca}^{2+}\right]$ (prior to mixing with EDTA) higher than $25 \mu \mathrm{M}$, they observe biexponential dissociations of calcium from Fluo-3 (with rates around $200 \mathrm{~s}^{-1}$ and 600 $\mathrm{s}^{-1}$ ) whereas for initial $\left[\mathrm{Ca}^{2+}\right]$ below $10 \mu \mathrm{M}$ the dissociation is monoexponential with a rate of $424 \mathrm{~s}^{-1}$ at $\mathrm{pH} 7.40,22^{\circ} \mathrm{C}$. Working with calcium levels within the dynamic range of the dye, we only see monoexponential relaxations which provide us with independent estimates of on- and off-rates. Ellis-Davies et al [36] have given an estimate for the off-rate of Calcium-Orange-5N (12 600 $\mathrm{s}^{-1}$ ) based on fitting the fluorescence increase of CalciumOrange $-5 \mathrm{~N}$ upon laser photolysis of calcium cages with a complex kinetic system. We present a direct determination of both, $k_{-1}$ and $k_{1}$, which does not rely on photolysis rates and binding rates of calcium to other compounds in a complex scheme.

Furthermore, we measured the on-rates of calcium binding to EGTA and H-EDTA to be $2.7 \times 10^{6} \mathrm{M}^{-1} \mathrm{~s}^{-1}$ and $4.5 \times 10^{6} \mathrm{M}^{-1} \mathrm{~s}^{-1}$, respectively, at $\mathrm{pH} 7.20$. In case of ECTA, several studies have used stopped-flow techniques $[17,18]$ and arrived at values between $1.0-3.5 \times 10^{6} \mathrm{M}^{-1} \mathrm{~s}^{-1}$ around neutral $\mathrm{pH}$, however under buffer conditions and ionic strengths which do not match our patch clamp conditions. So, we decided to also reinvestigate EGTA using the same method like the other chelators. With the T-jump approach, we arrive at the same number for EGTA. Hence, the HEPES buffer and the presence of 140 $\mathrm{mM} \mathrm{CsCl}$ do not significantly affect the kinetics. In addition, the $\mathrm{pH}$ dependence is not very strong in the range between 6.8 and 7.20 . Only at higher $\mathrm{pH}$, the acid groups of EGTA are deprotonated which strongly speeds up the association kinetics of calcium. The H-EDTA kinetics are quite similar to EGTA providing us with a low-affinity, slow calcium buffer. On the other hand, DPTA appears to be a fast, low-affinity buffer. Actually, we cannot give precise values for its on- and off-rate because we are lacking a sufficiently fast, low affinity calcium indicator. Here, an analogue of the longwavelength indicators will be very useful with on-rate around $5 \times 10^{8} \mathrm{M}^{-1} \mathrm{~s}^{-1}$ and $\mathrm{K}_{\mathrm{D}}$ bigger than $500 \mu \mathrm{M}$. Unfortunately, such an indicator for very fast measurements (relaxation times in the $\mu$ s range) is not yet available. But in future, with the advent of faster imaging technologies, it could prove to be a very powerful tool in studying rapidly developing and dissipating calcium microdomains close to the mouth of a calcium channel. To get a decent signal, a prerequisite would be a good fluorescence increase upon calcium binding. Here, we give lower bounds for the on- and off-rate of DPTA, based on the observations of its relaxation with CalciumGreen $-5 \mathrm{~N}$ and the theoretically expected relaxation rates in this case. If the on-rate was smaller than $4.0 \times 10^{7}$ $\mathrm{M}^{-1} \mathrm{~S}^{-1}$, we could deduce its value with the help of Calcium-Green $-5 \mathrm{~N}$ as a sufficiently fast reporter. Since the kinetics of Calcium-Green-5N dictate the fluorescence relaxation, the on-rate must be bigger than the above value.

Wc have presented in vitro kinctic measurements emphasizing that our interest is to quantitatively study fast, calcium-mediated physiological processes in vivo. Thus, the question immediately arises whether we can transfer these data to in vivo situations. In a recent study [37], flash photolysis of caged calcium was used to deduce the association rate constant of Fura- 2 and the endogenous calcium buffer from observations of fluorescence relaxation in bovine adrenal chromaffin cells. The estimated Fura- 2 on-rate in these 'calciumjump' experiments is $5 \times 10^{8} \mathrm{M}^{-1} \mathrm{~s}^{-1}$ which is in very good agreement with in vitro data. Similarly, the authors give an in vivo calcium association rate of DM-nitrophen (3.5 $\left.\times 10^{7} \mathrm{M}^{-1} \mathrm{~s}^{-1}\right)$ which is comparable with other in vitro estimates $\left(8 \times 10^{7} \mathrm{M}^{-1} \mathrm{~s}^{-1}\right.$ according to [36]). Thus, in bovine adrenal chromaffin cells, the in vivo kinetics, to the extent to which they have been studied so far, seem to match the in vitro kinetics. The situation, however, appears to be different in skeletal muscle fibers. Baylor and his colleagues $[6,31,32,38]$ have systematically 
investigated the impact of the myoplasmic environment on the affinities of different indicators. They conclude that the $K_{\mathrm{D}}$ values can be increased by a factor of 3 or more. Furthermore, from studies of fluorescence transients in response to an action potential, they give values for $\mathbf{k}_{-1}$ and, with this and $\mathrm{K}_{\mathrm{p}}$, give an upper value for the on-rate of Calcium-Orange-5N $\left(<1.2 \times 10^{7} \mathrm{M}^{-1} \mathrm{~s}^{-1}\right)$. This number is an order of magnitude smaller than ours. One explanation can be the binding of the dye to unknown myoplasmic constituents which alter the kinetics. In fact, they estimate between $50-90 \%$ of the indicator to be bound to unknown cellular components. Contrasting these type of data with the chromaffin data and our T-jump data, the question remains whether the myoplasm constitutes a very specific kinetic environment which is somehow different from other cells' cytoplasm. There is only one way to answer this question: performing in vivo kinetic relaxation measurements in different cells. This is the work which needs to be done in future.

\section{ACKNOWLEDGEMENTS}

I would like to thank Dr Erwin Neher for his constant support during the course of this study and many insightful discussions. I am also thankful to Drs Hartmuth Diebler and Dietmar Poerschke for help on instrumentation and helpful discussions.

\section{REFERENCES}

1. Grynkiewicz G., Poenie M., Isien R.Y. A new generation of $\mathrm{Ca}^{2+}$ indicators with greatly improved fluorescence properties. J Biol Chem 1985; 260: 3440-3450.

2. Minta A., Kao J.P.Y., Tsien R.Y. Fluorescent indicators for cytosolic calcium based on rhodamine and fluorescein chromophores. J Biol Chem 1989; 264: 8171-8178.

3. Kaplan J.H. Photochemical manipulation of divalent cation levels. Annu Rev Physiol 1990; 52: 897-914.

4. Neher E., Zucker R.S. Multiple calcium-dependent processes related to secretion in bovine chromaffin cells. Neuron 1993; 10: $21-30$.

5. Thomas P., Wong J.G., Lee A.K., Almers W. A low affinity $\mathrm{Ca}^{2+}$ receptor controls the final steps in peptide secretion from pituitary melanotrophs. Neuron 1993; 11: 93-104.

6. Zhao M., Hollingworth S., Baylor S.M. Properties of tri- and tetracarboxylate $\mathrm{Ca}^{2+}$ indicators in frog skeletal muscle fibers. Biophys J 1996; 70: 896-916.

7. Zucker R.S. Calcium and transmitter release. J Physiol Paris 1993; 87: 25-36.

8. Kao J.P.Y., Tsien R.Y. Calcium binding kinetics of fura- 2 and azo-1 from temperature-jump relaxation measurements. Biophys J 1988; 53: 635-640.

9. Eigen M., DeMaeyer L. Techniques of Organic Chemistry. New York: Wiley, 1963

10. Bernasconi C.F. Relaxation Kinetics. New York: Academic Press, 1976.

11. Rigler R., Rabl C.R., Jovin T.M. A temperature-jump apparatus for fluorescence measurements. Rev Sci Instrum 1974; 45: $580-588$.
12. Acton F.S. Numerical Methods that Work. New York: Harper \& Row, 1970.

13. Neher E., Augustine G.J. Calcium gradients and buffers in bovine chromaffin cells. J Physiol 1992; 450: 273-301.

14. Tsien R.Y. New calcium indicators and buffers with high selectivity against magnesium and protons: design, synthesis, and properties of prototype structures. Biochemistry 1980; 19 : 2396-2404.

15. Heinemann C., Chow R.H., Neher E., Zucker R.S. Kinetics of the secretory response in bovine chromaffin cells following flash photolysis of caged $\mathrm{Ca}^{2+}$. Biophys $J$ 1994; 67: 2546-2557.

16. Pörschke D. Cable temperature jump apparatus with improved sensitivity and time resolution. Rev Sci Instrum 1976; 47: 1363-1365.

17. Smith P.D., Liesegang G.W., Berger R.L., Czerlinski G., Podolsky R.J. Stopped-flow investigation of calcium ion binding by ethylene glycol bis(beta-aminoethyl ether)-N,N'-tetraacetic acid. Anal Biochem 1984; 143: 188-195.

18. Harafuji H., Ogawa Y. Re-examination of the apparent binding constant of ethylene glycol bis(beta-aminoethyl ether)$\mathrm{N}, \mathrm{N}, \mathrm{N}^{\prime}, \mathrm{N}^{\prime}$-tetraacetic acid with calcium around neutral $\mathrm{pH} . J$ Biochem 1980; 87: 1305-1312.

19. Stanley E.F. Single calcium channels and acetylcholine release at a presynaptic nerve terminal. Neuron 1993; 11: 1007-1011.

20. Llinas R., Sugimori M., Silver R.B. The concept of calcium concentration microdomains in synaptic transmission. Neuropharmacology 1995; 34: 1443-1451.

21. Chad J.E., Eckert R. Calcium 'domains' associated with individual channels may account for anomalous voltage relations of $\mathrm{Ca}$ dependent responses. Biophys J 1984; 45: 993-999.

22. Llinas R.R., Steinberg I.Z., Walton K. Relationship between presynaptic calcium current and postsynaptic potential in squid giant synapse. Biophys $J$ 1981; 33: 323-351.

23. Heidelberger R., Heinemann C., Neher E., Matthews G. Calcium dependence of the rate of exocytosis in a synaptic terminal. Nature 1994; 371: 513-515.

24. Borst J.G., Sakmann B. Calcium influx and transmitter release in a fast CNS synapse. Nature 1996; 383: 431-434.

25. Imredy J.P., Yue D.T. Submicroscopic $\mathrm{Ca}^{2+}$ diffusion mediates inhibitory coupling between individual $\mathrm{Ca}^{2+}$ channels. Neuron 1992; 9: 197-207.

26. Cooper D.M.F., Mons N., Karpen J.W. Adenylyl cyclases and the interaction between calcium and cAMP signalling. Nature 1995; 374: 421-424.

27. Roberts W.M. Localization of calcium signals by a mobile calcium buffer in frog saccular hair cells. $J$ Neurosci $1994 ; 14$ : 3246-3262.

28. Wu Y-C., Tucker T., Fettiplace R. A theoretical study of calcium microdomains in turtle hair cells. Biophys J 1996; 71: 2256-2275.

29. Neher E. Concentration profiles of intracellular calcium in the presence of a diffusible chelator. In: Heinemann U., Klee M., Neher E., Singer W. (Eds). Calcium Electrogenesis and Neuronal Functioning. Berlin: Springer, 1986; 80-96.

30. Stern M.D. Buffering of calcium in the vicinity of a channel pore. Cell Calcium 1992; 13: 183-192.

31. Konishi M., Hollingworth S., Baylor S.M. Myoplasmic calcium transients in intact frog twitch fibers monitored with furaptra, Mag-Fura-2 and purpurate diacetic acid Pdaa [Abstract: ThirtyFourth Annual Meeting of the Biophysical Society]. Biophys J 1990; 57 : 344a

32. Konishi M., Hollingworth S., Baylor S.M. Calcium transients in frog skeletal muscle fibers measured with the fluorescent magnesium indicator Mag-Fura-2. [Abstract: Forty-Third Annual Meeting of the Society of General Physiologists]. J Gen Physiol 1989; 94: 13a-14a. 
33. Eberhard M., Erne P. Calcium binding to fluorescent calcium indicators: calcium green, calcium orange and calcium crimson. Biochem Biophys Res Commun 1991; 180: 209-215.

34. Eberhard M., Erne P. Kinetics of calcium binding to fluo-3 determined by stopped-flow fluorescence. Biochem Biophys Res Commun 1989; 163: 309-314.

35. Lattanzio F.A., Bartschat D.K. The effect of $\mathrm{pH}$ on rate constants, ion selectivity and thermodynamic properties of fluorescent calcium and magnesium indicators. Biochem Biophys Res Commun 1991; 177: 184-191.
36. Ellis-Davies G.C.R., Kaplan J.H., Barsotti R.J. Laser photolysis of caged calcium: rates of calcium release by nitrophenyl-EGTA and DM-nitrophen. Biophys $J$ 1996; 70: 1006-1016.

37. Xu T., Naraghi M., Kang H., Neher E. Kinetic studies of $\mathrm{Ca}^{2+}$ binding and $\mathrm{Ca}^{2+}$ clearance in the cytosol of adrenal chromaffin cells. Biophys J 1997; 73: 532-545.

38. Baylor S.M, Hollingworth S., Konishi M. Calcium transients in intact frog single skeletal muscle fibers measured with the fluorescence indicator dye mag-fura- 2 . Meeting of The Physiological Society, Cambridge, UK, July 1989.

\section{APPENDIX}

In the experiments which we described in the main body of this paper, we always had two different calcium buffers present. Let us call them A and B, respectively. Since they both compete for binding to calcium, we are given the following kinetic system:

$$
\begin{aligned}
& \mathrm{Ca}^{2+}+\mathrm{A} \underset{\mathrm{k}_{-\mathrm{A}}}{\stackrel{\mathrm{k}_{\mathrm{A}}}{\rightleftarrows}} \mathrm{CaA} \\
& \mathrm{Ca}^{2+}+\mathrm{B} \underset{\mathrm{K}_{-B}}{\stackrel{\mathrm{K}_{B}}{\rightleftarrows}} \mathrm{CaB}
\end{aligned}
$$

where $k_{A}, k_{-A}, k_{B}$ and $k_{-B}$ are the binding and unbinding rate constants of $A$ and $B$, respectively. If the total concentrations of $A$ and $B$ are fixed during an experiment, i.e. $[A]_{T} \equiv[A]+[\mathrm{CaA}]$ and $[B]_{\mathrm{T}} \equiv[\mathrm{B}]+[\mathrm{CaB}]$ are constants, the following rate equations are valid at any point in time - assuming spatial homogeneity of the concentrations in our sample:

$$
\begin{aligned}
& \frac{d x_{1}}{d t}=-k_{A} \cdot x_{1} \cdot\left([A]_{T}-x_{2}\right)+k_{-A} \cdot x_{2}-k_{B} \cdot x_{1} \cdot\left([B]_{T}-x_{3}\right)+k_{-B} \cdot x_{3} \\
& \frac{d x_{2}}{d t}=k_{A} \cdot x_{1} \cdot\left([A]_{T}-x_{2}\right)-k_{-A} \cdot x_{2} \\
& \frac{d x_{3}}{d t}=k_{B} \cdot x_{1} \cdot\left([B]_{T}-x_{3}\right)-k_{B} \cdot x_{3}
\end{aligned}
$$

where $\mathrm{x}_{1}=\left[\mathrm{Ca}^{2+}\right], \mathrm{x}_{2}=[\mathrm{CaA}]$ and $\mathrm{x}_{3}=[\mathrm{CaB}]$. Let $\mathrm{x}$ denote the instantaneous concentration vector $\mathrm{x}_{2}=\left(\mathrm{x}_{1} \mathrm{x}_{2} \mathrm{x}_{3}\right)^{\mathrm{t}}, \overline{\mathrm{x}}$ some equilibrium concentration which is temperature dependent and $\delta x=x-\bar{x}$ the concentration deviation from equilibrium. The system (Equations $A 2-A 4)$ is a vector field equation which can be abbreviated as $\left.d x / d t=f\left(x,[A]_{T}, B\right]_{T}\right)$ with $f$ representing the righthand side of the above equations. For small deviations from equilibrium, standard Taylor series expansion permits a linearization of the above vector field around $\bar{x}$, giving rise to $f(x) \approx f(\bar{x})+D f(x) \cdot(x-\bar{x})$, and hence

$$
\dot{\delta} \mathrm{x}=\mathrm{Df}(\overline{\mathrm{x}}) \cdot \delta \mathrm{x} \quad \text { Eq. } \mathrm{A} 5
$$

with $\operatorname{Df}(\overline{\mathrm{x}})$ being the matrix of partial derivatives of $\mathrm{f}$ at the equilibrium point $\overline{\mathrm{x}}$. It is easy to see that, using the abbreviations $\mathrm{a}_{1}=-\mathrm{k}_{\mathrm{A}} \cdot\left([\mathrm{A}]_{\mathrm{T}}-\overline{\mathrm{x}}_{2}\right), \mathrm{a}_{2}=-\mathrm{k}_{\mathrm{B}} \cdot\left([\mathrm{B}]_{\mathrm{T}}-\overline{\mathrm{x}}_{3}\right), \mathrm{a}_{3}=\mathrm{k}_{-\mathrm{B}}+\mathrm{k}_{\mathrm{B}} \cdot \overline{\mathrm{x}}_{1}$ and $\mathrm{a}_{4}=\mathrm{k}_{-\mathrm{A}}+\mathrm{k}_{\mathrm{A}} \cdot \overline{\mathrm{x}}_{1}$, we get:

$$
D f(\bar{x})=\left[\begin{array}{ccc}
a_{1}+a_{2} & a_{3} & a_{4} \\
-a_{2} & -a_{3} & 0 \\
-a_{1} & 0 & -a_{4}
\end{array}\right]
$$

If we change the temperature of the solution in a stepwise fashion, i.e. rapid compared to the mean times for the reaction of $\mathrm{A}$ and $\mathrm{B}$ with calcium, at time $\mathrm{t}=0$ and denote by $\overline{\mathrm{x}}$ the concentration vector after the temperature jump, 
then the system has to relax from the initial value $x_{0} \equiv \mathbf{x}(t=0) \neq \overline{\mathbf{x}}$ towards $\overline{\mathbf{x}}$ according to the dynamic system given by Equation A5. The general solution to Equation $A 5$ is given by the sum of two exponentials plus a dc offset, i.e.

$$
\delta x=\sum_{i=1}^{2} \alpha_{i} \cdot e^{-\lambda_{1}, t} \cdot u_{i}+\alpha_{0} \cdot u_{0}
$$

where $\alpha_{i}$ are some real numbers incorporating the initial condition and $u_{i}$ some vectors. The rates $\lambda_{i}$ are the absolute values of the nonzero eigenvalues of the matrix and can easily be seen to be given by:

$$
\lambda_{1 / 2}=\frac{a_{1}+a_{2}-a_{3}-a_{4} \pm \sqrt{\left(a_{1}+a_{2}-a_{3}-a_{4}\right)^{2}-4 \cdot\left(a_{3} \cdot a_{4}-a_{2} \cdot a_{4}-a_{1} \cdot a_{3}\right)}}{2}
$$

Re-arranging the sums in the above expression and noticing that $a_{1}-a_{4}=-k_{A} \cdot\left(\left[\mathrm{Ca}^{2+}\right]+[A]\right)-k_{-A} \equiv-\lambda_{A}$ is the rate for the relaxation of

$$
\mathrm{Ca}^{2+}+\mathrm{A} \underset{\mathrm{k}_{-\mathrm{A}}}{\stackrel{\mathrm{k}_{\mathrm{A}}}{\rightleftarrows}} \mathrm{CaA}
$$

and, similarly, $a_{2}-a_{3}=-k_{B} \cdot\left(\left[\mathrm{Ca}^{2+}\right]+[B]\right)-k_{-B} \equiv-\lambda_{B}$ the rate for the relaxation of

$$
\mathrm{Ca}^{2+}+\mathrm{B} \underset{\mathrm{k}_{\mathrm{B}}}{\stackrel{\mathrm{k}_{\mathrm{B}}}{\rightleftarrows}} \mathrm{CaB}
$$

we end up with the following formula for the absolute values of the relaxation rates of the system (Eq. A1):

$$
\lambda_{1 / 2}=\frac{\left.\left.\lambda_{A}+\lambda_{B} \pm \sqrt{\left(\lambda_{A}+\lambda_{B}\right)^{2}-4 \cdot k_{B} \cdot\left[\lambda_{A} \cdot\left(\mathrm{K}_{B}+\left[\overline{\mathrm{Ca}^{2+}}\right]\right)+k_{A} \cdot \overline{[B}\right]\left(K_{A}+\overline{\left[\mathrm{Ca}^{2+}\right.}\right]}\right)\right\}}{2}
$$

Here

$$
K_{A}=\frac{k_{-A}}{k_{A}} \text { and } K_{B}=\frac{k_{-B}}{k_{B}}
$$

are the dissociation constants of the two buffers A and B, respectively. In the Results section, we used certain limiting cases of Equation $\mathrm{A} 9$ to fit the measured relaxation rates to the theoretically expected ones. 